\title{
Cell therapy for macular degeneration-first phase I/II pluripotent stem cell-based clinical trial shows promise
}

\author{
CHEN ZhiGuo ${ }^{1,2^{*}}$ \& ZHANG Y. Alex ${ }^{1,2^{*}}$ \\ ${ }^{1}$ Cell Therapy Center, Xuanwu Hospital, Capital Medical University, and Key Laboratory of Neurodegeneration, Ministry of Education, \\ Beijing 100053, China; \\ ${ }^{2}$ Center of Neural Injury and Repair, Beijing Institute for Brain Disorders, Beijing 100069, China
}

Received November 20, 2014; accepted November 28, 2014; published online December 18, 2014

Citation: Chen ZG, Zhang YA. Cell therapy for macular degeneration-first phase I/II pluripotent stem cell-based clinical trial shows promise. Sci China Life Sci, 2015, 58: 119-120, doi: 10.1007/s11427-014-4791-2

Embryonic stem (ES) cells, a type of pluripotent stem cells (PSCs) that are derived from the inner cell mass of blastocysts, have been discovered for more than three decades [1]. Given the almost infinite self-renewal capacity and the potential to differentiate into any specific cell type in an individual, ES cells and PSCs have brought hope for the treatment of many unmet diseases. However, the advantages of PSCs - the highly proliferative and plastic capacities, also give rise to concerns regarding the development of tumors and differentiation of undesirable cell types, when engrafted into a human being. These concerns have hampered the application of PSCs in clinics and not until 2010 was the first clinical trial using PSC-derived cells approved in the world. The long awaited medium- to long-term results of the trials that came out in a recent Lancet publication confirmed that hESC-derived cells improved vision in macular degeneration patients while showing no apparent safety concerns [2], bringing much relief to some and considerable excitement to many in the stem cell field.

Macular degeneration is the leading cause of blindness in developed countries, which involves degeneration of retinal pigment epithelial (RPE) cells leading to photoreceptor loss and deterioration of visual acuity. Because eyes are immune-privileged organ and require only a relatively small dose of cells to show efficacy, macular degeneration has become the first-in-human clinical trial approved that in-

*Corresponding author (email: chenzhiguo@gmail.com; yaz@bjsap.org) volves the use of PSCs. In 2010, the US Food and Drug Administration approved the application of ES cells-derived RPE cells for subretinal delivery to treat macular degeneration in humans and the first patient was enrolled in 2011 (ClinicalTrials.gov, numbers NCT01345006 and NCT01344993).

The short-term follow-up results from two registered patients were published in Lancet in 2012 [3]. And the recent Lancet publication provided the medium- and long-term follow-up results with 18 enrolled patients, including nine dry age-related macular degeneration and nine Stargardt's macular dystrophy patients [2]. The nine patients of either disease were further divided into three groups, with each group receiving 50,000, 100,000, and 150,000 RPE cells, respectively. The RPE cells were differentiated from ES cells using a previously established protocol, which yielded homogeneous RPE cells of more than $99 \%$ purity. Prior to transplantation, the best-corrected visual acuity in the study eyes ranged from 20/200 (severe vision loss) to hand motion (near blindness). For patients in both study groups, treated eyes have shown more improvement from baseline as compared to the untreated eyes. In total, eight of the 18 patients experienced an increase in visual acuity of at least 15 letters one year after transplantation, which corresponds to doubling of the visual angle, and is generally accepted as a clinically significant improvement. By using a sensitive and reliable Visual Function Questionnaire, trained examiners measured the vision-targeted quality of life for the 
patients before and at various time points after transplantation. For age-related macular degeneration, the mental health and vision subscales for general vision, peripheral vision, near activities, and distance activities improved from baseline by a median of 16-25 points 3-12 months after transplantation. For patients with Stargardt's macular dystrophy, there was an improvement of 8-20 points 3-12 months after transplantation.

Compared to the efficacy of improving vision, more attention was probably paid to the safety profile of the trials, in particular, whether there was any indication of tumor formation. The median follow-up time in the reported study was 22 months, with the longest one up to 37 months. With this medium- to long-term follow-up time, no safety issues related to the engrafted cells were observed. No occurrences of adverse proliferation, growth of transplanted tissue at the injection site, or differentiation to ectopic tissue/cells (non-RPE cells) was observed. No sign of acute transplant rejection was noted in any of the operated eyes. Although a small extent of preretinal, pigmented cell growth was seen in three eyes near the injection site, no adverse effects were caused by these small epiretinal pigmented cell patches, which was considered to be a visually insignificant event. Several systemic adverse events observed in patients seem to be related to the regimen of immune suppression, which started from one week before and through 12 weeks after transplantation.

The improvement of visual acuity may not be conclusively approved because of the inherent caveats of open-label trials, which, as the authors have discussed, may include the possibilities of sham-surgery rescue effect, placebo effect, and improvement due to repeated testing and intersession variability. However, the current trial has provided quite solid ground for the safety of this interventional strategy, which itself is a major step forward in stem celltherapy involving PSCs.
As noted above, some complications observed in the study were actually associated with the immunosuppression regimen. The side effects were particularly obvious in elderly patients. Another source of PSCs - induced pluripotent stem cells (iPSCs) may circumvent such problems. iPSCs can be derived from patients' own somatic cells and possess almost identical genomic background [4]. In July 2013, Japan approved the first clinical trial using iPS cells-derived RPE cells for treatment of macular degeneration, which was led by ophthalmologist Masayo Takahashi at the RIKEN Center for Developmental Biology (CDB) in Kobe, Japan. On September 12, 2014, the first woman received transplantation of iPSC-derived RPE cells to treat macular degeneration.

Stem cell therapy is a rapidly progressing field. If ESand/or iPSC-derived RPE cells are proved to be safe and efficacious for treatment of macular degeneration, it will have a significant impact on the regulatory bodies in other nations and accelerate the clinical translation of cell therapy for other diseases, such as Parkinson's disease and diabetes.

1 Evans MJ, Kaufman MH. Establishment in culture of pluripotential cells from mouse embryos. Nature, 1981, 292: 154-156

2 Schwartz SD, Regillo CD, Lam BL, Eliott D, Rosenfeld PJ, Gregori NZ, Hubschman JP, Davis JL, Heilwell G, Spirn M, Maguire J, Gay R, Bateman J, Ostrick RM, Morris D, Vincent M, Anglade E, Del Priore LV, Lanza R. Human embryonic stem cell-derived retinal pigment epithelium in patients with age-related macular degeneration and Stargardt's macular dystrophy: follow-up of two open-label phase 1/2 studies. Lancet advance online publication 15 October 2014; doi:10.1016/S0140-6736(14)61376-3

3 Schwartz SD, Hubschman JP, Heilwell G, Franco-Cardenas V, Pan CK, Ostrick RM, Mickunas E, Gay R, Klimanskaya I, Lanza R. Embryonic stem cell trials for macular degeneration: a preliminary report. Lancet, 2012, 379: 713-720

4 Takahashi K, Yamanaka S. Induction of pluripotent stem cells from mouse embryonic and adult fibroblast cultures by defined factors. Cell, 2006, 126: 663-676

Open Access This article is distributed under the terms of the Creative Commons Attribution License which permits any use, distribution, and reproduction in any medium, provided the original author(s) and source are credited. 